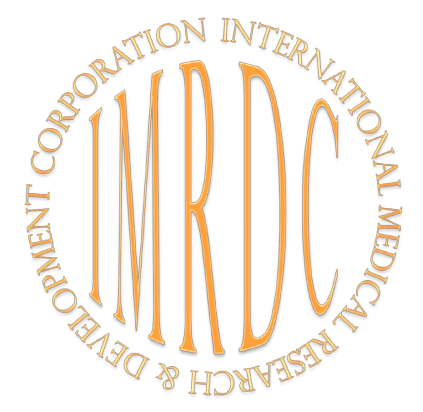

\title{
Health Relationship Management Services (HRMS) A New Healthcare Paradigm Using the 5Rs
}

\author{
Nik Tehrani, PhD \\ DynoSense Academy, CA, USA
}

\begin{abstract}
Health Relationship Management Services (HRMS) is a new paradigm comprised of the 5Rs which define a comprehensive patient healthcare system in the digital health age. This service uses a health monitoring system with sensors and a patient application portal that can Read data and securely send it to the cloud with amended data from Electronic Health Records (EHR). The read data is Restructured by analytics software for evaluation and personalization in an aggregated form represented by an individualized health scoring and demographic grading system. The Results are previously restructured data that must become actionable to lead to an escalation path to the Communication Center for a Response. On a periodic basis, Repeat of the cycle will improve patient health outcomes through an interconnected link of services for optimal continuous health relationship management. Providers can leverage the HRMS platform to generate data-driven approaches fostering a positive and lasting impact on their patient's health. Payers can track data to ensure a more healthy patient population and review meaningful outcomes. (Int J Biomed. 2016;6(1):87-89.).
\end{abstract}

Keywords: Health Relationship Management Services (HRMS); new health paradigm; 5Rs; Patient Health Narratives (PHN); Patient Engagement.

\section{Health Relationship Management Services (HRMS)}

Digital health has been quite successful in monitoring patients' health and, according to a latest finding, U.S. investments in digital health have reached $\$ 2.3$ billion for the first six months of this year, which has broken all past records [1]. Healthcare, today, is one of the sectors that is most influenced by advanced technology. Developments in medical technology have progressed from digital wristbands that measure heart rates and other health data to a new generation of products that are improving healthcare delivery and outcomes [2] by enabling providers to remain continuously connected with their patients no matter where they are. These innovative systems can indicate a patient's adherence to protocols and, in some cases, may even act as an early-warning system for “degenerative illnesses such as Alzheimer's and Parkinson's disease" [2]. High-tech sensors can now "monitor the at-home cardiac patient's heart every minute of every day" without being hooked up to a monitor [2]. Similar to something out of science fiction, new technological devices now can remotely

*Corresponding author: Nik Tehrani, PhD. DynoSense Academy, San Jose, CA, USA. E-mail:ntehrani@dynosense.com scan a human body to detect a myriad of symptoms, with the results available to patients, physicians, care providers and relatives. The patient data that is read by the sensors is more valuable than an intermittent "snapshot" [2] [3] because it is uninterrupted and it is sent instantaneously to the cloud for restructuring by analytics. For instance, Steinhubl is working on a "continuous blood pressure measurement watch" to monitor blood pressure over time, not just when a patient is at the doctor's office. According to Steinhubl, there is a lack of "synchronicity - there are asynchronous appointments for doctor visits, and so on; so most of healthcare hasn't really been designed around the patient to make sure their blood pressure, lipids, diabetes, are well-controlled." "Patient care has become a primary focus in the development of new concepts and knowledge in healthcare technology" [4]. To improve patient care, the health sector is using data and technology [5]. The core idea is that digital technology ought to be planned to help manage patients more efficiently and that manufacturers should provide, if need be through strategic alliances, more than just a device by also offering patient healthcare services and solutions in one coordinated platform.

The current paradigm of medical care relies on the "autonomous and highly trained doctor" [6] to gather and manage information required for every patient's care. This paradigm is confronted by (i) the accumulation of obligations 
for knowledge by patients and physicians; (ii) by the demand to evaluate patients not part of a physician's practice; (iii) "by consistently unmet quality of care expectations; (iv) by the expense of outmoded, fragmented, and suboptimal care; (v) and by a seemingly insurmountable demand for chronic disease care" [6]. Refinements within the old paradigm of medical care may not solve such challenges, suggesting the need for a new paradigm [6]. There is a need for a unified definition of digital healthcare, or connected health, in which everyone can speak the same language.

Health Relationship Management Services (HRMS) is this new paradigm which defines comprehensive patient healthcare in the digital health age for individualized patient care, engagement, and managed outcomes. An obvious benefit of this technology paradigm is the generation of quality clinical data, since up until recently, was raw data that may not have been transferred to a care provider in a way it could be actionable [4] [7]. HRMS is a revolutionary new definition to place all digital health activities and technologies within one cohesive cyclical system where patient data is collected from variety of sources, analyzed, made actionable, acted upon, and repeated. HRMS is a complete health ecosystem that produces positive outcomes as a comprehensive service, much as having a physician at home monitoring the patient on a daily basis to minimize health risk while reducing cost of care.

An example of one part of HRMS is a health monitoring system that can measure physiological parameters such as ECG, blood pressure, respiration, heart rate, and other vitals to safeguard a patient's good health. This digital healthcare comes in the forms of small devices or limited smartphone applications. HRMS works on the principle of five services known as the 5Rs, which are Read, Restructure, Results, Response, and Repeat. Metaphorically speaking, these services are "turning gears," with the first one turning, causing the next one to turn, and so on, as one related collaborative routine (Figure 1). Read turns the Restructure, which turns Results, which turns Response, and Repeat completes and enhances the overall service.

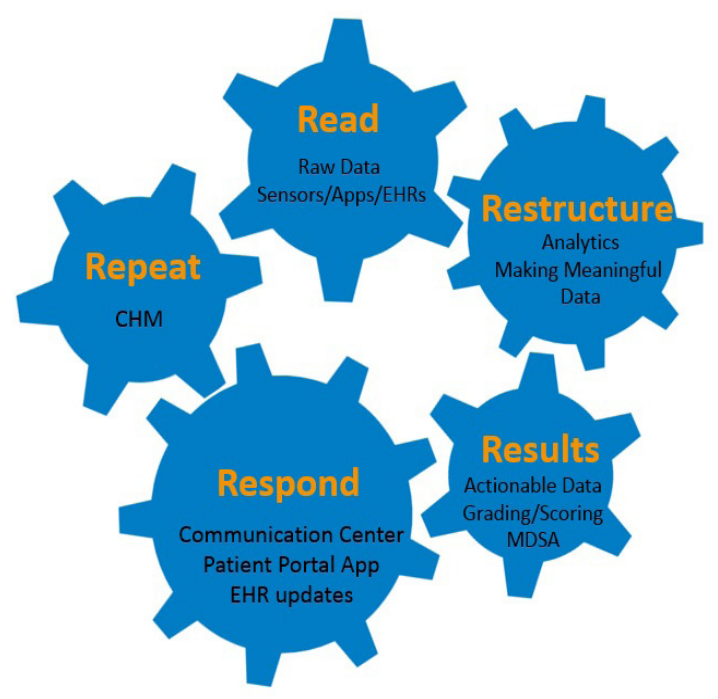

Fig. 1. Health Relationship Management Services represented as "gears", with each turning the other in sequence for a continuous cycle.

\section{Read}

Read is defined as compilation of raw captured data from a variety of sources such as: (i) a health monitoring system with sensors, (ii) an interactive patient application portal, (iii) Electronic Health Records (EHR), (iv) care specialist feedback, and (v) care friends responses. Read data is securely sent to the cloud to ensure patients' health data privacy.

An example of a health monitoring system is the "Dyno ${ }^{\mathrm{TM}}$ " from DynoSense Corp., [8] which is able to measure, at home, various health metrics such as ECG, heart rate, blood $\mathrm{O}_{2}$, respiration rate, breathing efficiency, blood pressure change, body temperature, breath gases, and body compositions. Taking readings efficiently depends on the quality of the sensors and simplicity of usage. Furthermore, the patient portal, DynoLife ${ }^{\mathrm{TM}}$, interactively engages the patient for psychological and behavioral feedback among a community of care experts and care friends. Read data are stored in the cloud and are ready to be restructured as required [9].

\section{Restructure}

Read patient data stored in the cloud is a raw format where it is required to be Restructured by analytics software for evaluation and personalization. This analyzed data in an aggregated form is represented by an individualized health scoring and demographic grading system. Restructure of the data is a vital step as it involves the data analysis and interpretation of the measurement obtained. There can be many ways to interpret the readings, but the best systems are based on proven medical standards identified by sources, such as the AMA (American Medical Association). By a solid interpretation, HRMS is able to convert the acquired reading into valuable and meaningful data, not just an accumulation of meaningless Big Data, which can be used for an overall evaluation of the patient.

\section{Results}

Once the restructuring has been completed, actionable Results must be generated for proper response through an escalation path which indicates all of the individual's significant health data. This is exhibited by examples such as (i) individualized health scoring and demographic grading; (ii) Health Indexing (HI), which captures health data over a period of time to create better understanding of the true health condition of the individual; (iii) Medical Decision Support Algorithm (MDSA ${ }^{\mathrm{TM}}$ ), which filters unnecessary data for the physicians and care providers; and (iv) medication effectiveness. The validity of this system can be cross-checked to confirm if the results indicate a health risk that the care specialist and/or care provider can verify.

\section{Response}

From the results, a series of actions are identified that are required to be further qualified by the Response system. Such a quantifying process takes many forms, such as (i) 
interactive questionnaires or surveys through the patient portal application platform, (ii) text or email messaging, (iii) automated robocalls, and (iv) live video or voice interactions with a health specialist from the Communication Center. Following the quantifying process, an appropriate response is developed. Examples of appropriate responses are (i) escalation to a care specialist for further intervention, (ii) scheduling a clinic or hospital visit, (iii) Emergency room visit, (iv) entry into EHR/EMR records of the individual, or (v) updated individualized status for further personalized activities. Activities may include (i) news feeds with upto-the minute health information, (ii) gamification, which is health recognition games with a reward system to encourage [10] (iii) and an interactive online magazine encouraging individuals to share their Patient Health Narratives (PHN) and become a member of a healthcare community [11]. Patient Health Narratives (PHN) is an emergent trend of providing a platform for patients to share their health related stories and dialogues. This delivers a voice to the experiences of illness, to avoid feelings of isolation, and alleviate concerns and fears of chronic conditions and medical procedures. This interactive patient application portal may create a sense of community and support for the patient.

\section{Repeat}

Repeat is the Continuous Health Management (CHM) of an individual's status passing through the entire cycle. On a periodic basis, Repeat of the cycle will improve patient health outcomes through an interconnected link of services for optimal continuous health relationship management. Individuals are empowered by this continuous process not only by the up-to-the minute availability of their health information, but also, in a meaningful and actionable process, they become part of the customized social health community of care experts and care friends. The HRMS platform can be leveraged to produce data-driven approaches to advance a positive and long-term influence on their patient's health. Data can be tracked by payers to assure a more healthy patient population and to review meaningful outcomes.

\section{Conclusion}

Health Relationship Management Services (HRMS) works on the principle of five services known as the 5Rs, which are Read, Restructure, Results, Response, and Repeat. The combined 5Rs complete the cycle of HRMS through a continuous cyclic HRMS process in that one element is dependent upon the others. Through patient engagement, the individual becomes part of the solution with continuous personalized feedback, encouragement, empowerment, inspiration, and inclusion in a health community that ultimately may lead to lifestyle changes.

HRMS was conceived to define and standardize the entire health ecosystem with continuous follow-up and feedback to the patient, providers, and payers. As companies adopt the entire patient experience of HRMS, patients will receive health services as a product. Health related costs will decrease, especially for providers visits while improving quality of care.

\section{References}

1. Health. (2015). Digital Health Funding Soars To Record \$2.3 Billion, But Crowdfunding Tumbles - See more at: http:// nuviun.com/content/digital-health-funding-soars-to-record23-billion-but-crowdfunding-tumbles\#sthash.3vxkm3LZ. dpuf. Retrieved from http://nuviun.com/: http://nuviun.com/ content/digital-health-funding-soars-to-record-23-billion-butcrowdfunding-tumbles

2. Morrisey J. (2015). The medical technologies that are changing health care. New, eye-popping medical technology provides earlier diagnoses, personalized treatments and a breathtaking range of other benefits for both patients and health care professionals. Retrieved from HYPERLINK "http://www. hhnmag.com/articles/3580-the-medical-technologies-that-arechanging-health-care" http://www.hhnmag.com/articles/3580the-medical-technologies-that-are-changing-health-care

3. Steinhubl S. (2016). Steven Steinhubl, M.D., director of digital medicine at the Scripps Translational Science Institute, shares his perspectives on the current opportunities in mobile health. IHT2. Institute for health technology transformation. Retrieved from http://ihealthtran.com/wordpress/2014/01/ steven-steinhubl-md-director-of-digital-medicine-scrippstranslational-science-institute-clinical-cardiologist-scrippshealth/

4. Cassano C. (2014). The right balance -technology and patient care. OJNI Volume 18, Number 3. Retrieved from http://www.himss.org/ResourceLibrary/GenResourceDetail. aspx?ItemNumber $=33541$

5. PWC. (2015). Healthcare and innovation. Retrieved from http://www.pwc.com/us/en/health-industries/top-healthindustry-issues/healthcare-technology-and-innovation.html

6. White. (2008). Health information technology will shift the medical care paradigm. J Gen Intern Med. 2008 Apr;23(4):495-9. doi: 10.1007/s11606-007-0394-y.

7. Schmittdiel J. (2015). Using technology to improve health. research scientists study the intersection of technology and health. Kaiser permanente. Retrieved from https:// www.dor.kaiser.org/external/DORExternal/research_report/ research technology.aspx

8. Tehrani N, Meckl-Sloan C. Remote health monitoring in action. International Journal of Business Management and Economic Research (IJBMER). 2015;6(1):111-4.

9. Lupton D. (2013). Donna Haraway: The digital cyborg assemblage and the new digital health technologies. The Palgrave handbook of social theory in health, illness and medicine, 567-581.

10. Merriam-Webster. (2015). Gamification. Retrieved from http://www.merriam-webster.com/dictionary/gamification 11. Meckl-Sloan C. (2015). A new healthcare paradigm: patient health narratives: enhancing patient engagement through sharing stories. Retrieved from http://www.gjar.org/ publishpaper/vol2issue9/d288r70.pdf 